Bài báo khoa học

\title{
Giám sát biến thiên mức độ phú dưỡng của hồ Hoàn Kiếm dựa vào hàm lượng Chlorophyll-a tính toán từ ảnh Sentinel-2A
}

\author{
Nguyễn Thiên Phương Thảo ${ }^{1}$, Phạm Quang Vinh², Nguyễn Thị Thu Hà ${ }^{1, *}$, Nguyễn Thùy \\ $\operatorname{Linh}^{1,3}$ \\ ${ }^{1}$ Khoa Địa chất, Trường Đại học Khoa học Tự nhiên, Đại học Quốc gia Hà Nội; \\ nguyenthienphuongthao_t57@hus.edu.vn \\ ${ }^{2}$ Viện Địa lý, Viện Hàn lâm Khoa học và Công nghệ Việt Nam; pqvinh@ig.vast.vn \\ ${ }^{3}$ Khoa Địa lý, Trường Đại học Khoa học Tự nhiên, Đại học Quốc gia Hà Nội; \\ nguyenthuylinh@hus.edu.vn \\ * Tác giả liên hệ: hantt_kdc@vnu.edu.vn; Tel.: +84-2435587062
}

Ban Biên tập nhận bài: 12/11/2020; Ngày phản biện xong: 04/12/2020; Ngày đăng bài: $25 / 01 / 2021$

Tóm tắt: Nghiên cứu này nhằm giám sát sự dao động của chỉ số dinh dưỡng (trophic state index: TSI) của nước hồ Hoàn Kiếm dựa vào hàm lượng chlorophyll-a (Chla) tính toán từ ảnh vệ tinh Sentinel 2A (S2A) thu nhận được từ tháng 9 năm 2019 đến tháng 8 năm 2020. Kết quả khảo sát từ 50 điểm đo tại hồ Hoàn Kiếm vào 5 thời điểm khác biệt trong năm cho thấy hàm lượng Chla của nước hồ dao động trong khoảng $114,8 \mu \mathrm{g} / \mathrm{L}$ đến hơn $700 \mu \mathrm{g} / \mathrm{L}$ và có tương quan cao với tỷ số phản xạ mặt nước ứng với kênh cận hồng ngoại (B5) trên kênh đỏ $(\mathrm{B} 4)$ của ảnh $\mathrm{S} 2 \mathrm{~A}\left(\mathrm{R}^{2}=0,82\right)$ do đó có thể tính toán từ tỷ số này bằng phương trình hàm mũ với sai số trung bình đã kiểm chứng là $29,4 \mu \mathrm{g} / \mathrm{L}$. Giá trị TSI(Chla) tính toán từ ảnh và thực tế cho giá trị khá tương đồng, dao động ở mức từ 77 đến 95 tương ứng với mức siêu phú dưỡng, cho thấy tiềm năng cao của việc giám sát mức độ phú dưỡng của hồ từ ảnh $\mathrm{S} 2 \mathrm{~A}$. Theo không gian, giá trị TSI(Chla) thay đổi không nhiều, thường tập trung ở mức cao xung quanh khu vực bờ phía bắc và phía nam. Giá trị TSI(Chla) tính toán từ ảnh $\mathrm{S} 2 \mathrm{~A}$ có sự thay đổi nhẹ theo mùa, cao vào đầu hè (tháng 6 ) và đầu đông (tháng 10,12 ). Để giám sát được chi tiết hơn biến thiên giá trị TSI(Chla) cần khai thác thêm các vệ tinh khác như Landsat 8 và Sentinel $2 \mathrm{~B}$ để tăng tần suất giám sát.

Từ khóa: Phú dưỡng; Hồ Gươm; Chlorophyll-a; TSI; Sentinel 2A.

\section{Mở đầu}

Úng dụng công nghệ viễn thám trong đánh giá chất lượng nước đã được ứng dụng rộng rãi và đạt được nhiều thành tựu quan trọng trong hơn 40 năm qua [1-4]. Hầu hết các nghiên cứu đều được xây dựng và phát triển dựa trên mối quan hệ giữa đặc tính quang học của nước với các thông số chất lượng nước như tổng chất rắn lơ lửng, độ sâu thấu quang hay hàm lượng Chlorophyll-a (Chla) [5].

Hiện nay, tình trạng phú dưỡng đang xảy ra thường xuyên và được đánh giá là một trong những mối đe dọa hàng đầu đến các hệ sinh thái thủy sinh ở các hồ đô thị trên khắp thế giới [6]. Cũng như các hồ đô thị khác trên thế giới, các hồ đô thị ở Hà Nội hiện nay đang đứng trước nguy cơ suy thoái do đô thị hóa, biến đồi khí hậu, ô nhiễm và đặc biệt là hiện tượng phú dưỡng. Hồ Hoàn Kiếm là một trong những hồ nước ngọt tự nhiên có vai trò đặc biệt quan trọng trong đời sống văn hóa-chính trị của người dân Hà Nội, là biểu tượng của thành phố 
cũng đang phải đối mặt với tình trạng phú dưỡng nghiêm trọng, đây là nhận định được đưa ra dựa trên việc xác định hàm lượng các chất dinh dưỡng như Phốtpho tổng số và Nitơ tổng số trong nước [7], cũng như mật độ tảo đo được [8]. Do đó, việc phát triển và ứng dụng các kỹ thuật định lượng vào giám sát hiện tượng phú dưỡng của hồ Hoàn Kiếm là vô cùng cần thiết để quản lý môi trường nước hồ cũng như duy trì đa dạng sinh học, bảo tồn các loài gen quý.

Đã có nhiều nghiên cứu tiến hành đánh giá tình trạng phú dưỡng của các hồ đô thị, trong đó phổ biến nhất là sử dụng chỉ số trạng thái phú dưỡng (TSI-Trophic State Index). [9] đã đề xuất tính toán TSI của hồ thông qua 1 trong 3 đại lượng: độ sâu thấu quang, hàm lượng Chla và Phốtpho tổng số. Trong số các đại lượng trên, hàm lượng Chla hay còn gọi chất diệp lục là thông số chính phản ánh thực vật phù du trong nước và thường được sử dụng để đánh giá mức độ phú dưỡng của thủy vực [10] vì nó phản ánh trực tiếp sức khỏe của hệ sinh thái thủy sinh hơn là các chỉ số thứ cấp như tỉ số của Nitơ tổng số với Phốtpho tổng số. Ú̉ng dụng công nghệ viễn thám để tính toán hàm lượng Chla là một trong những ứng dụng cơ bản nhất được sử dụng phổ biến trong giám sát chất lượng nước [11], do vậy, trong nghiên cứu này, Chla được lựa chọn là thông số dùng đánh giá mức độ phú dưỡng của Hồ Hoàn Kiếm bằng phương pháp viễn thám. Đã có rất nhiều thuật toán sử dụng các kênh phổ riêng lẻ hay tỷ số các kênh phổ phản xạ từ các dữ liệu vệ tinh đa phổ khác nhau để tính toán Chla trong nước [12], điển hình như thuật toán dựa trên tỷ lệ kênh phổ xanh lục/xanh lam [13-14], xanh lục/đỏ [15-16] hay như tỷ lệ kênh phổ cận hồng ngoại (NIR)/đỏ [17-18] áp dụng vào vùng nước ven biển hay hồ nội địa. Tuy nhiên, Hồ Hoàn Kiếm là một hồ có điều kiện môi trường nước rất đặc trưng với nồng độ sinh khối cao, giá trị Chla thường ở mức từ $129 \mu \mathrm{g} / \mathrm{L}$ dến hơn $500 \mu \mathrm{g} / \mathrm{L}$ dẫn đến tình trạng xuất hiện liên tục hiện tượng "tảo nở hoa" [19] nên việc xây dựng thuật toán tính toán Chla từ các dữ liệu vệ tinh còn nhiều tồn tại. Đặc biệt, do kích thước của hồ Hoàn Kiếm tương đối nhỏ nên các dữ liệu ảnh vệ tinh sử dụng cần hội tụ đủ sự phù hợp của cả hai yếu tố: độ phân giải không gian và độ phân giải bức xạ.

Dữ liệu ảnh vệ tinh Sentinel $2 \mathrm{~A}(\mathrm{~S} 2 \mathrm{~A})$ của Cơ quan vũ trụ Châu Âu được đánh giá là dữ liệu ưu việt để giám sát phú dưỡng các hồ đô thị với độ phân giải không gian cao $(10 \mathrm{~m})$, chu kỳ thu dữ liệu ngắn (10 ngày), miễn phí. Đặc biệt với thiết kế chuyên biệt cho vùng cận hồng ngoại (có nhiều điểm tương đồng với ảnh vệ tinh MERIS - vệ tinh được ESA phát triển trước đó để giám sát cho môi trường biển), $\mathrm{S} 2 \mathrm{~A}$ được đánh giá là một dữ liệu phù hợp để nghiên cứu, giám sát môi trường nước đô thị, đảm bảo tính khả thi và hiệu quả về mặt chi phí.

Nghiên cứu này nhằm đánh giá và giám sát mức độ phú dưỡng Hồ Hoàn Kiếm thông qua việc tính toán Chla và giá trị TSI(Chla) từ ảnh vệ tinh $\mathrm{S} 2 \mathrm{~A}$. Trong đó, phương trình tính toán Chla từ các thông số ảnh $\mathrm{S} 2 \mathrm{~A}$ được xây dựng dựa trên kết quả đo Chla và phổ phản xạ mặt nước đo cùng thời kỳ ở nhiều thời điểm khảo sát khác nhau. Phương trình này sau đó được áp dụng vào các ảnh S2A đã được xử lý để tính toán được giá trị TSI(Chla) của nước hồ làm cơ sở đánh giá mức độ phú dưỡng của hồ và sự thay đổi mức độ phú dưỡng nước hồ theo thời gian.

\section{Phương pháp nghiên cứu}

\subsection{Phưong pháp lấy mẫu và đo phổ hiện trưòng}

Tổng số 50 điểm khảo sát được thực hiện tại hồ Hoàn Kiếm vào 3 đợt khảo sát: đợt 1 : ngày $14 / 04 / 2016,06 / 10 / 2016$ và ngày $26 / 10 / 2016$; đợt 2 : ngày $31 / 10 / 2018$; và đợt 3 : ngày 17/07/2020. Tại mỗi điểm khảo sát, nhóm nghiên cứu đều tiến hành đo phổ phản xạ $\left(\rho_{\mathrm{w}}\right)$, lấy mẫu nước để phân tích hàm lượng Chla trong phòng thí nghiệm và định vị bằng GPS (Hình 1).

Theo đó, phổ phản xạ mặt nước được đo bằng máy đo bức xạ hiện trường GER1500 có dải phổ từ $350 \mathrm{~nm}$ đến $1050 \mathrm{~nm}$ với độ phân giải kênh phổ là $1,5 \mathrm{~nm}$, theo phương pháp của Mobley [20], trong đó sự ảnh hưởng của hiện tượng lấp lánh mặt nước được hiệu chỉnh dựa 
vào sự loại trừ phổ phản xạ của bầu trời. Phổ phản xạ mặt nước sau đó được chuẩn hóa về phổ phản xạ ứng với các kênh phổ của ảnh S2A theo phương pháp của Baris [21].

Cùng với đó, các mẫu nước mặt hồ Hoàn Kiếm được lấy ở độ sâu từ $0-50 \mathrm{~cm}$ và đựng trong chai sạch, sẫm màu với dung tích 1 lít, sau đó được bảo quản lạnh và vận chuyển đến phòng thí nghiệm. Trong phòng thí nghiệm, hàm lượng Chla được xác định dựa theo phương pháp tiêu chuẩn SMEWH 10200H:2012 của APHA [22].
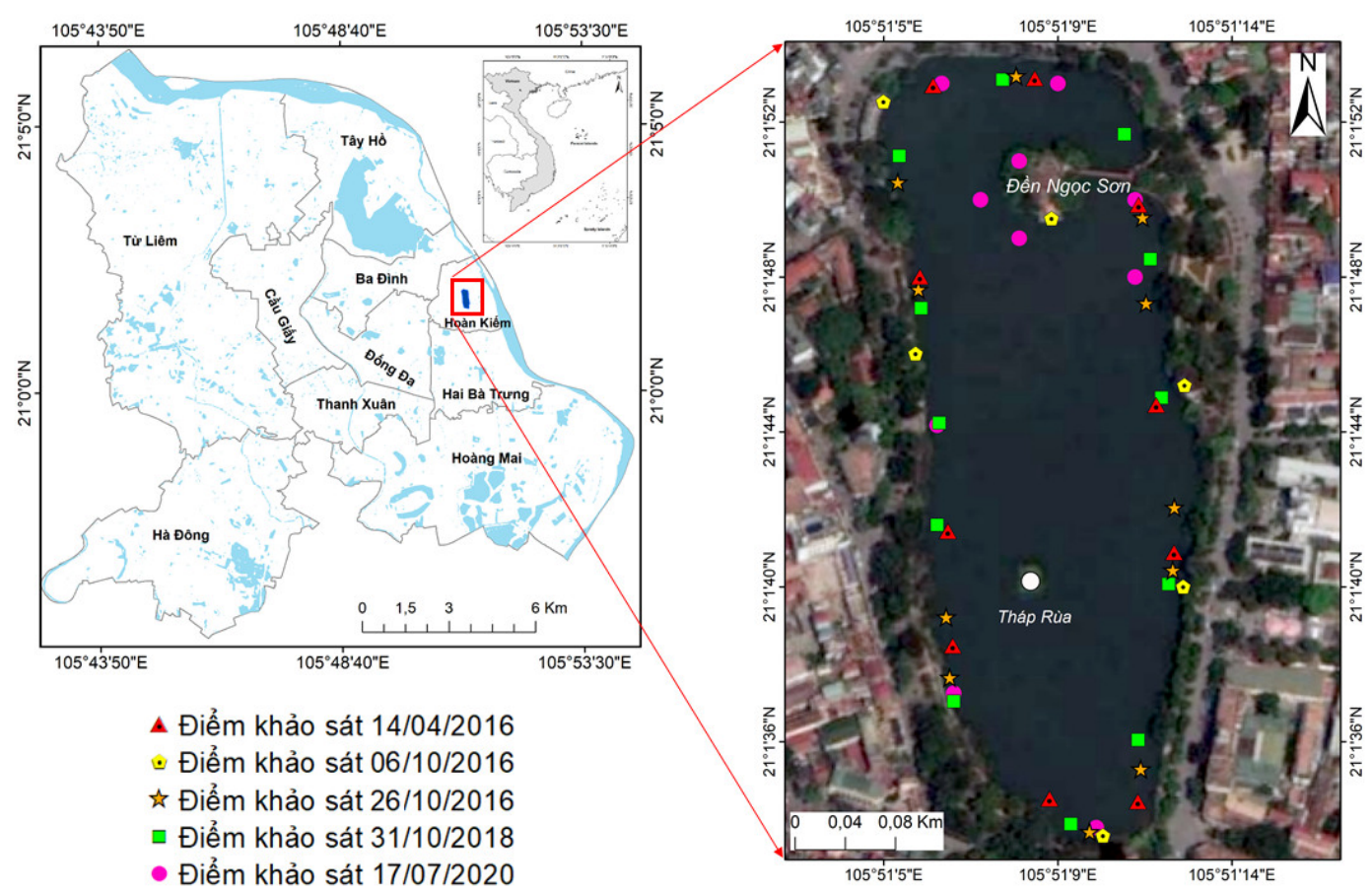

Hình 1. Vị trí Hồ Hoàn Kiếm tron các điểm khảo sát hồ Hoàn Kiếm trong 5 đợt khảo sát trên nền ảnh vệ tinh GeoEyes thu được ngày 05/08/2020.

Cuối cùng, mức độ phú dưỡng của nước hồ được xác định thông qua TSI được tính toán từ Chla theo công thức [23], cụ thể như sau:

$$
\operatorname{TSI}(\text { Chla })=9,81 * \ln (\text { Chla })+30,6
$$

Trong đó TSI(Chla) là đại lượng không thứ nguyên, Chla có đơn vị là $\mu \mathrm{g} / \mathrm{L}$. Dựa vào giá trị TSI(Chla), trạng thái phú dưỡng của hồ được chia thành 4 mức độ: 1) nghèo dinh dưỡng (oligotrophy, TSI(Chla) < 30); 2) dinh dưỡng trung bình (mesotrophy, TSI(Chla) $=30$ đến 50); 3) phú dưỡng (eutrophy, TSI(Chla) = 50 đến 70); 4) siêu phú dưỡng (hypereutrophy, TSI(Chla) > 70).

\subsection{Phương pháp xủ lý ảnh vệ tinh}

Vệ tinh S2A chụp ảnh khu vực Hà Nội vào khoảng 3:20 GMT (tương ứng với 10:20 giờ địa phương) trong hệ tọa độ WGS84, múi 48N với độ phân giải không gian là $10 \mathrm{~m}, 30 \mathrm{~m}$ và $60 \mathrm{~m}$. Trong nghiên cứu này, 10 cảnh ảnh S2A thu được vào các thời điểm khác nhau, có độ che phủ mây dưới $10 \%$ được tải miễn phí từ trang web của ESA (https://scihub.copernicus.eu/dhus/) được sử dụng (Bảng 1).

Tất cả các cảnh ảnh sử dụng đã được hiệu chỉnh bức xạ và hiệu chỉnh khí quyển để đưa dữ liệu về giá trị phản xạ tại mặt đất (BOA-reflectance) bởi ESA trước khi đến tay người sử dụng. Do vậy, trong nghiên cứu này, phương pháp xử lý ảnh chủ yếu là hiệu chỉnh hình học. Theo đó, các cảnh ảnh được đồng nhất độ phân giải không gian về $10 \mathrm{~m}$ sử dụng công cụ Resampling trong phần mềm SNAP (Sentinel Application Platform). Dữ liệu mặt nước sau 
đó được chiết tách và tính toán TSI(Chla) dựa vào các công cụ tính toán của phần mềm ENVI 5.3 .

Bảng 1. Các ảnh vệ tinh sử dụng trong nghiên cứu.

\begin{tabular}{clcc}
\hline STT & Các ảnh vệ tinh sử dụng trong nghiên cứu & Ngày \\
\hline 1 & S2A_MSIL2A_20190921T032541_N0213_R018_T48QWJ_20190921T073132 & $21 / 09 / 2019$ \\
2 & S2A_MSIL2A_20191001T032541_N0213_R018_T48QWJ_20191002T155146 & $01 / 10 / 2019$ \\
3 & S2A_MSIL2A_20191210T033131_N0213_R018_T48QWJ_20191210T070124 & $10 / 12 / 2019$ \\
4 & S2A_MSIL2A_20200218T032811_N0214_R018_T48QWJ_20200218T072701 & $18 / 02 / 2020$ \\
5 & S2A_MSIL2A_20200309T032551_N0214_R018_T48QWJ_20200309T073920 & $09 / 03 / 2020$ \\
6 & S2A_MSIL2A_20200428T032541_N0214_R018_T48QWJ_20200428T073032 & $28 / 04 / 2020$ \\
7 & S2A_MSIL2A_20200518T032541_N0214_R018_T48QWJ_20200518T073303 & $18 / 05 / 2020$ \\
8 & S2A_MSIL2A_20200627T032541_N0214_R018_T48QWJ_20200627T073329 & $27 / 06 / 2020$ \\
9 & S2A_MSIL2A_20200717T032541_N0214_R018_T48QWJ_20200717T072805 & $17 / 07 / 2020$ \\
10 & S2A_MSIL2A_20200826T032541_N0214_R018_T48QWJ_20200826T073212 & 26/08/2020 \\
\hline
\end{tabular}

\subsection{Phương pháp phân tích thống kê và bản đồ}

Các phép phân tích hồi quy, thống kê cơ bản, tính toán độ lệch, sai số trong nghiên cứu được thực hiện bằng phần mềm SPSS Statistics 20. Hệ số tương quan sử dụng trong bài báo là hệ số Pearson. Các phép phân tích đều dựa trên $95 \%$ phân bố của các chuỗi số.

Bản đồ phân bố không gian của TSI(Chla) được thành lập dựa trên phương pháp phân bố xác suất của biến ngẫu nhiên sử dụng modul phân mảnh mật độ (density slicing) trong ENVI 5.3 và biên tập trong ArcGIS 10.3.

\section{Kết quả và thảo luận}

\section{1. Đặc trung môi trường nước hồ Hoàn Kiếm}

Kết quả phân tích hàm lượng Chla trong nước hồ Hoàn Kiếm cho thấy hàm lượng Chla dao động trong khoảng từ $114,8 \mu \mathrm{g} / \mathrm{L}$ cho đến hơn $700 \mu \mathrm{g} / \mathrm{L}$, trung bình khoảng $259 \mu \mathrm{g} / \mathrm{L}$, độ lệch chuẩn tại mỗi điểm lên đến $121,1 \mu \mathrm{g} / \mathrm{L}$ (Hình $2 \mathrm{~A})$. Theo đó, giá trị TSI(Chla) thực tế của nước hồ Hoàn Kiếm dao động từ 77 đến 95 , trung bình đạt 84 (Hình 2B). Như vậy, dựa theo thang đánh giá mức độ phú dưỡng của hồ đề xuất bởi [23] thì nước hồ tại những điểm đo ở các thời điểm khác nhau đều ứng với mức siêu phú dưỡng - mức phú dưỡng cao nhất, nhạy cảm với hiện tượng tảo nở hoa [23]. Theo thời gian, giá trị TSI(Chla) của hồ Hoàn Kiếm đạt mức cao nhất là 95 vào tháng 7 năm 2020 .
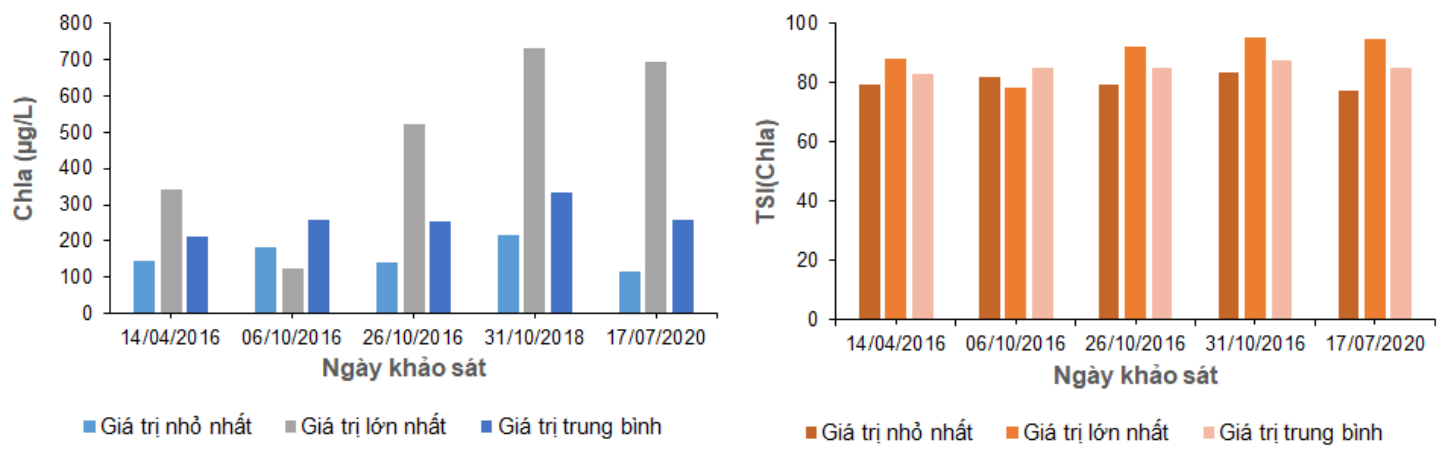

Hình 2. A) Kết quả đo hàm lượng Chla của nước hồ Hoàn Kiếm trong 5 lần khảo sát; B) Giá trị TSI(Chla) tính toán tương ứng với hàm lượng Chla đo được. 
Hình 3 thể hiện phổ phản xạ của mặt nước hồ Hoàn Kiếm tại mỗi điểm đo. Theo đó, tất cả các đường cong phổ cho thấy độ phản xạ cực đại của nước hồ nằm trong dải sóng từ 426$479 \mathrm{~nm}$ và $670-683 \mathrm{~nm}$ với 4 điểm cực đại lần lượt tương ứng ở các bước sóng từ 558-562 nm, 643-645 nm, 713-715 nm, và 804-807 nm. Dựa trên vị trí phân bố của các kênh phổ ảnh S2A (Hình 3), các kênh phổ ứng với điểm cực đại của phổ phản xạ là kênh 3 (xanh lục) và kênh 5 (cận hồng ngoại), trong khi kênh 1 (xanh lục) và kênh 4 (đỏ) gần với điểm cực tiểu của phổ phản xạ mặt nước.

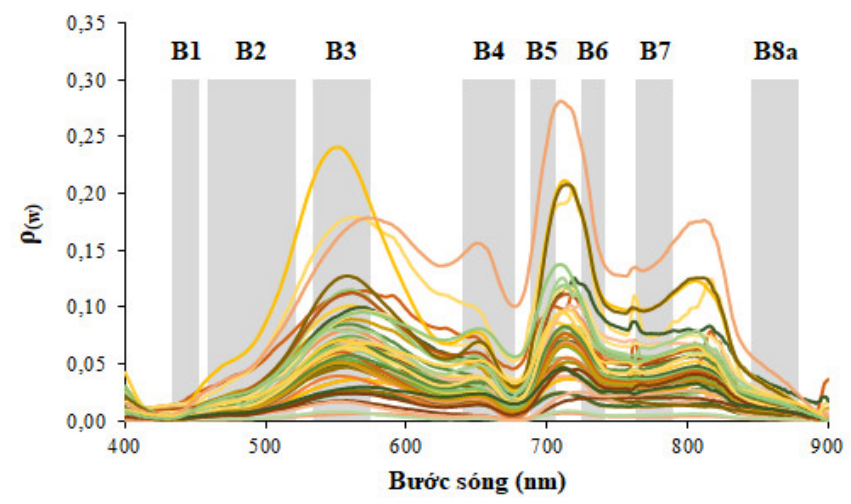

Hình 3. Đặc trưng phổ phản xạ mặt nước $\left(\rho_{\mathrm{w}}\right)$ đo tại hồ Hoàn Kiếm vào 5 lần khảo sát so với vị trí các kênh phổ ảnh S2A. B1 đến B8a ứng với vị trí các kênh phổ của ảnh S2A từ 1 đến 8 a.

\subsection{Mối quan hê giũa hàm lương Chla, TSI(Chla) và phổ phản xạ mặt nước}

Việc lựa chọn thành công các mô hình đơn kênh phổ hay các tỷ lệ kênh phổ để tính toán Chla phụ thuộc phần lớn vào các đặc tính hóa sinh của nước mà các đặc tính này được thể hiện rất rõ qua phổ phản xạ của nước. Trong nghiên cứu này, các thuật toán phổ biến thường dùng để ước tính hàm lượng Chla trong vùng nước siêu phú dưỡng như thuật toán dựa trên tỷ lệ dải phổ xanh lục/đỏ, cận hồng ngoại/đỏ hay xanh lam/xanh lục [11] sẽ được phân tích và đánh giá dựa trên dữ liệu của 38 điểm đo trong 4 ngày khảo sát. Kết quả phân tích bước đầu cho thấy, trong tất cả các trường hợp, phương trình đường cong có giá trị tương quan cao và sai số nhỏ nhất là đường cong hàm mũ (Bảng 2 ). Điều này một lần nữa khẳng định sự phù hợp của hàm số mũ để tính toán các thành phần chất lượng nước từ dữ liệu viễn thám đã được chứng minh trong nghiên cứu trước đó [24].

Bảng 2. Mối quan hệ tuyến tính giữa Chla và tỷ số phổ phản xạ ứng với dải phổ của kênh ảnh $\mathrm{S} 2 \mathrm{~A}$ sử dụng dữ liệu của 38 điểm đo trong 4 ngày khảo sát.

\begin{tabular}{|c|c|c|c|c|c|c|c|}
\hline \multirow[b]{2}{*}{ Thuật toán } & \multirow{2}{*}{$\begin{array}{c}\text { Tỷ lệ } \\
\text { kênh phổ } \\
\text { S2A }\end{array}$} & \multicolumn{2}{|c|}{ Hàm tuyến tính } & \multicolumn{2}{|c|}{ Hàm số mũ } & \multicolumn{2}{|c|}{ Hàm logarith } \\
\hline & & $\mathbf{R}^{2}$ & RMSE & $\mathbf{R}^{2}$ & RMSE & $\mathbf{R}^{2}$ & RMSE \\
\hline \multirow[t]{2}{*}{ Xanh lục/Đỏ } & $\mathrm{B} 3 / \mathrm{B} 4$ & 0,8 & 54,25 & 0,69 & 0,21 & 0,66 & 70,5 \\
\hline & B5/B4 & 0,86 & 45,30 & $\mathbf{0 , 8 2}$ & 0,17 & 0,75 & 60,6 \\
\hline \multirow{3}{*}{$\begin{array}{l}\text { Cận hồng } \\
\text { ngoại/Đỏ }\end{array}$} & B6/B4 & 0,78 & 56,53 & 0,68 & 0,22 & 0,73 & 63,7 \\
\hline & $\mathrm{B} 7 / \mathrm{B} 4$ & 0,77 & 58,33 & 0,65 & 0,23 & 0,68 & 69,18 \\
\hline & B8/B4 & 0,65 & 71,97 & 0,51 & 0,27 & 0,57 & 80,58 \\
\hline Xanh & $\mathrm{B} 3 / \mathrm{B} 1$ & 0,01 & 121,6 & 0,01 & 0,38 & 0,09 & 116,4 \\
\hline $\begin{array}{c}\text { lam/Xanh } \\
\text { lục }\end{array}$ & $\mathrm{B} 3 / \mathrm{B} 2$ & 0,08 & 117,4 & 0,02 & 0,38 & 0,1 & 116,1 \\
\hline
\end{tabular}


Trong nghiên cứu này, tỷ số kênh phổ cận hồng ngoại/đỏ (kênh 5/kênh 4) có giá trị tương quan ổn định và chặt chẽ hơn cả với hệ số xác định $\mathrm{R}^{2}=0,82, p=0,00$ và giá trị sai số của phép tính là $17 \mu \mathrm{g} / \mathrm{L}$ (Hình $4 \mathrm{a})$. Theo đó, hàm lượng Chla có thể tính toán tương đối chính xác từ ảnh $\mathrm{S} 2 \mathrm{~A}$ theo phương trình:

$$
\text { Chla }=101,58 \mathrm{e}^{0,27 \cdot \mathrm{B} 5 / \mathrm{B} 4}(\mu \mathrm{g} / \mathrm{L})
$$

Trong đó Chla là hàm lượng Chla trong nước hồ $(\mu \mathrm{g} / \mathrm{L})$; B5/B4 là tỷ số kênh $5 / \mathrm{kênh}$ 4 của ảnh S2A.
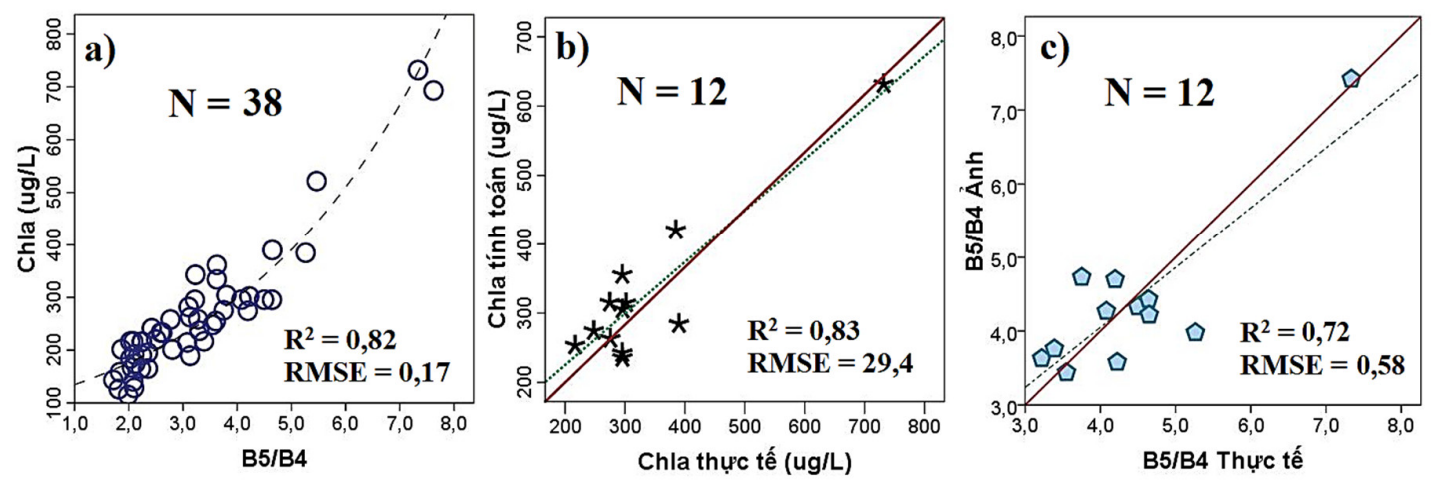

Hình 4. Biểu đồ biểu diễn: a) Mối quan hệ tuyến tính giữa hàm lượng Chla và tỷ số kênh $5 /$ kênh 4 (B5/B4) của ảnh $\mathrm{S} 2 \mathrm{~A}$; b) Sai số giữa Chla đo được thực tế và Chla tính toán từ phương trình hàm mũ hình $4 \mathrm{a}$ và $\mathrm{c})$ Sai số giữa phổ phản xạ $\mathrm{B} 5 / \mathrm{B} 4$ đo thực tế và phổ $\mathrm{B} 5 / \mathrm{B} 4$ thu hồi từ ảnh $\mathrm{S} 2 \mathrm{~A}$ của 12 điểm đo ngày 17/7/2020.

Để kiểm chứng mối quan hệ này, tập dữ liệu của 12 điểm đo thực tế thu vào ngày khảo sát 17/7/2020 được đưa vào đánh giá độ lệch chuẩn của kết quả tính toán từ phương trình (hình $4 b$ ). Kết quả cho thấy giá trị Chla tính toán từ phương trình (2) và giá trị đo thực tế có tương quan cao $\left(\mathrm{R}^{2}=0,83\right)$ và sai số $29,4 \mu \mathrm{g} / \mathrm{L}$ (dưới $10 \%$ giá trị trung bình của chuỗi số, Hình $4 \mathrm{~b}$ ). Kết quả này khẳng định tỷ số kênh $5 /$ kênh 4 của ảnh $\mathrm{S} 2 \mathrm{~A}$ hoàn toàn phù hợp để tính toán hàm lượng Chla của nước hồ Hoàn Kiếm nói riêng và môi trường nước nội địa siêu phú dưỡng nói chung đã được minh chứng trước đó [25].

Do phương trình (2) được xây dựng và kiểm chứng dựa trên giá trị phổ phản xạ mặt nước $\rho_{\mathrm{w}}$ và Chla đo đạc thực tế vào cùng thời điểm nên có độ chính xác gần như tuyệt đối vì không bị ảnh hưởng bởi các yếu tố thời tiết, khoảng cách hay các nhiễu động do các phản ứng, thành phần của bầu khí quyển như dữ liệu vệ tinh. Để áp dụng thành công phương trình (2) vào dữ liệu ảnh vệ tinh $\mathrm{S} 2 \mathrm{~A}$, phương pháp xử lý ảnh (cụ thể là phương pháp hiệu chỉnh khí quyển) để thu nhận phổ phản xạ mặt nước phải phù hợp và chính xác. Hình $4 \mathrm{c}$ biểu diễn sai số giữa tỷ số phổ phản xạ $\mathrm{B} 5 / \mathrm{B} 4$ thu được tại mặt nước và tỷ số phổ phản xạ thu được của $\mathrm{B} 5 / \mathrm{B} 4$ ảnh S2A Level 2A trong ngày 17/7/2020 tại 12 điểm đo ứng với 12 pixels ảnh. Kết quả cho thấy ảnh S2A Level 2A đã được hiệu chỉnh khí quyển khá phù hợp cho các vùng nước có độ phú dưỡng cao như nước hồ Hoàn Kiếm trong nghiên cứu này. Với sai số toàn phương trung bình là 0,58 , tương đương dưới $10 \%$ trung bình giá trị đo đạc thực tế; dữ liệu ảnh S2A Level $2 \mathrm{~A}$ này rất phù hợp cho việc tính toán các thông số nước bằng phương pháp sử dụng tỷ số kênh ảnh. Do đó, các kết quả tính toán Chla từ ảnh $\mathrm{S} 2 \mathrm{~A}$ Level $2 \mathrm{~A}$ và sử dụng phương trình (2) nói trên cho kết quả khá chính xác khi thay thế tỷ số B5/B4 của ảnh thay cho giá trị phổ mặt nước ứng với các kênh này.

Cũng từ mối quan hệ này, TSI(Chla) của nước hồ có thể tính toán từ ảnh S2A dựa trên sự kết hợp phương trình (1) và (2) như sau:

$$
\operatorname{TSI}(\text { Chla })=9,81 * \ln \left(101,58 \mathrm{e}^{0,27 \cdot \mathrm{B} 5 / \mathrm{B} 4}\right)+30,6
$$

Trong đó B5/B4 là tỷ số kênh 5/kênh 4 của ảnh S2A. 


\subsection{Mối quan hệ giữa hàm lượng Chla, TSI(Chla) và phổ phản xạ mặt nước}

Áp dụng phương trình (3) vào tính toán giá trị TSI(Chla) của nước hồ Hoàn Kiếm từ 10 cảnh ảnh vệ tinh S2A chụp hồ từ tháng 9 năm 2019 đến tháng 8 năm 2020, chúng tôi xây dựng được một chuỗi các bản đồ phân bố theo không gian và thời gian của TSI(Chla) nước hồ như trong hình 5. Dựa vào sự phân bố này có thể thấy, do diện tích hồ tương đối nhỏ nên sự chênh lệch về mặt không gian của TSI(Chla) không thể hiện rõ nét, đặc biệt vào các tháng có nhiệt độ không khí thấp như tháng 2 và tháng 3 . Sang đến tháng $4, \mathrm{TSI}(\mathrm{Chla})$ có giá trị $>$ 86 bắt đầu xuất hiện và tập trung chủ yếu về phía Đông Nam của đền Ngọc Sơn và phần ven bờ phía Nam gần tháp Rùa. Sang đến tháng 5 , giá trị TSI(Chla) $>90$ lại phân bố chủ yếu xung quanh phía Tây Bắc và Đông Nam của đền Ngọc Sơn. Xu hướng này càng thể hiện rõ nét trong tháng 6 năm 2020, khi giá trị TSI(Chla) > 90 luôn được ghi nhận tại các khu vực xung quanh đền Ngọc Sơn và phía Nam của Tháp Rùa. Giá trị TSI(Chla) vào các tháng 7,8 , 9 giảm nhẹ đồng đều khắp mặt hồ, luôn ở giá trị trên 80 , trong khi đó lại ở mức xấp xỉ cao hơn $85-86$ vào các tháng 10,12 .
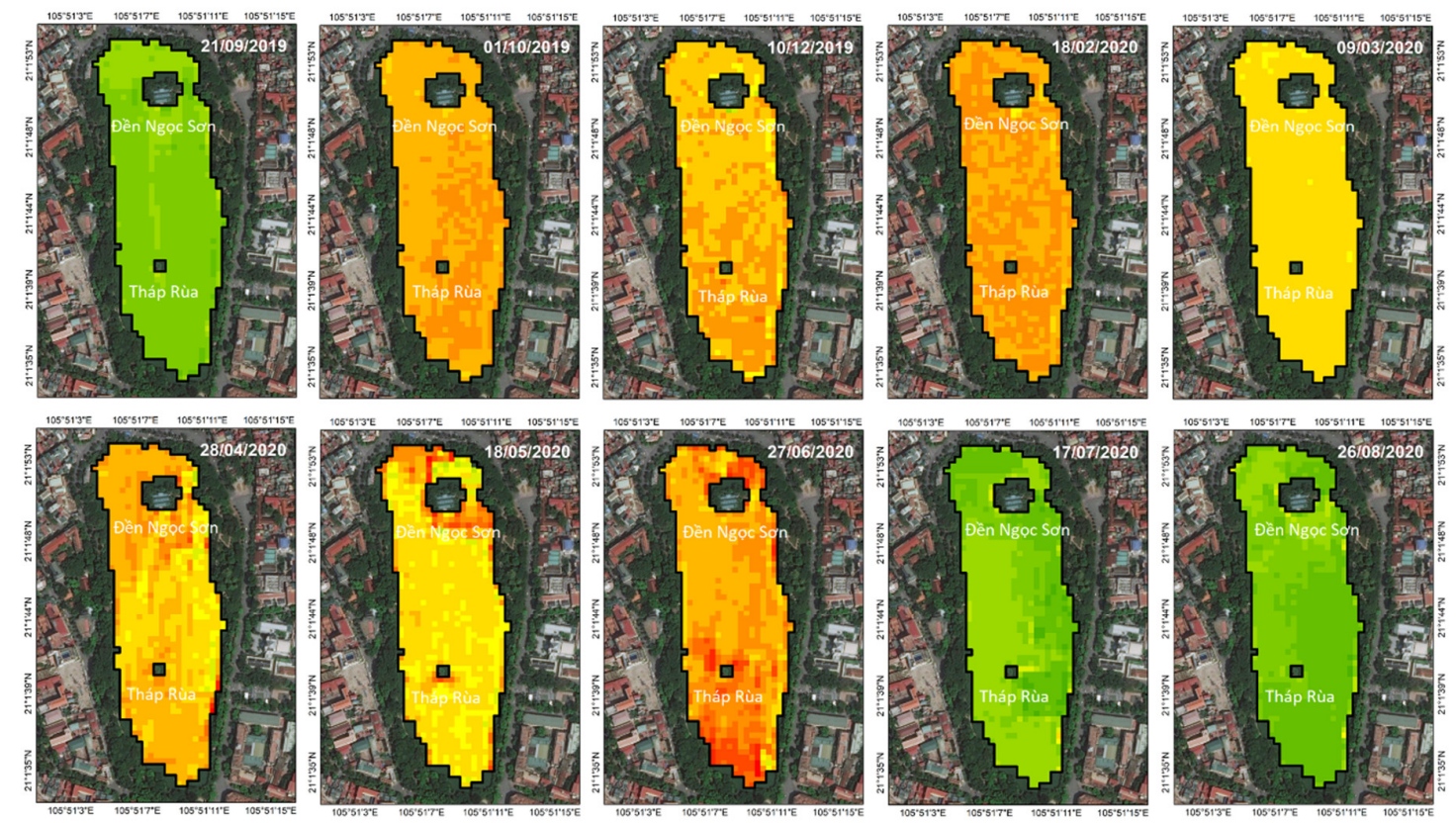

TSI (Chla)

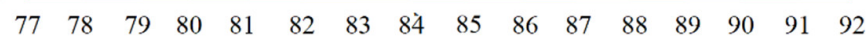

Hình 5. Phân bố TSI(Chla) của nước hồ Hoàn Kiếm từ tháng 9 năm 2019 đến tháng 8 năm 2020 .

Hình 6 biểu diễn sự thay đổi theo thời gian của giá trị TSI(Chla) tính toán từ 10 cảnh ảnh S2A thu được vào các thời điểm khác nhau trong năm 2019 và 2020 . Theo đó, giá trị TSI(Chla) trong tháng 2-3/2020 dao động trong khoảng từ 84-88 với giá trị trung bình đạt 85. Sang đến tháng 4 - giai đoạn chuyển mùa từ mừa khô sang mùa mưa, giá trị TSI(Chla) tăng dần nhưng không đáng kể với giá trị trung bình là 86 . Vào các tháng mùa mưa (tháng 5 đến tháng 10$)$, TSI(Chla) tăng dần dao động trong khoảng từ 78 và lên tới hơn 92 vào tháng 6. Sau đó, TSI(Chla) có xu hướng giảm dần trong tháng 7-9, sau đó tăng nhẹ cho đến tháng 1 năm sau. Dựa theo thang phân loại tình trạng phú dưỡng [23], trạng thái của hồ luôn ở mức siêu phú dưỡng, phù hợp với kết quả đo thực tế. 


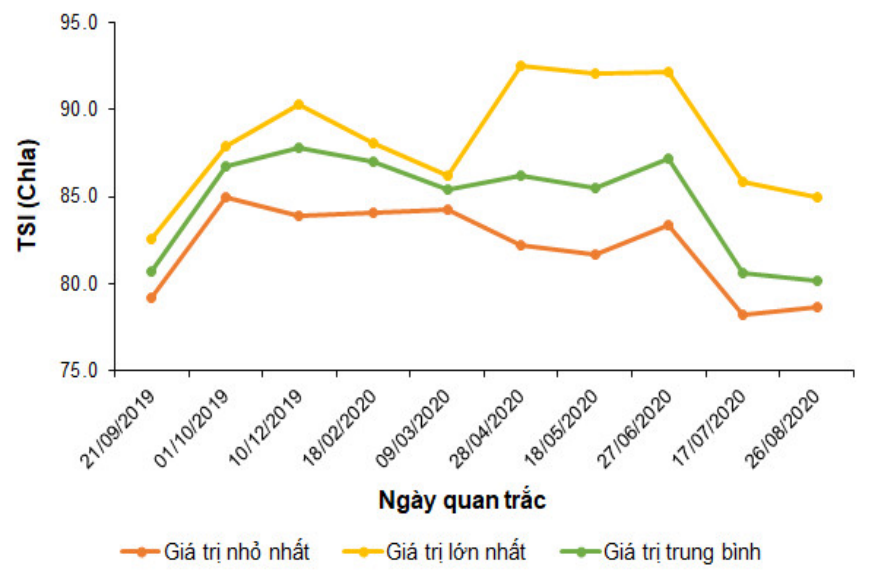

Hình 6. Sự thay đổi theo thời gian của giá trị TSI(Chla) của nước Hồ Hoàn Kiếm tính toán từ ảnh vệ tinh S2A thu được từ tháng 9 năm 2019 đến tháng 8 năm 2020.

\section{Kết luận}

Kết quả nghiên cứu cho thấy nước hồ Hoàn Kiếm trong khoảng thời gian từ tháng 9 năm 2019 đến tháng 8 năm 2020 luôn ở mức siêu phú dưỡng (hypertrophic) - mức phú dưỡng cao nhất có thể ảnh hưởng đến đời sống của hệ sinh thái thủy sinh $($ Chla $=114,8-700 \mu \mathrm{g} / \mathrm{L}$, $\mathrm{TSI}(\mathrm{Chla})=77$ đến 95$)$. Giá trị TSI của hồ có thể giám sát thông qua hàm lượng Chla tính toán từ ảnh $\mathrm{S} 2 \mathrm{~A}$ bằng hàm số mũ tuyến tính với biến là tỷ số kênh cận hồng ngoại (B5) trên kênh đỏ (B4) của ảnh. Giá trị TSI(Chla) thu được từ ảnh S2A có sự thay đổi nhẹ theo mùa và theo không gian hồ. Cụ thể, TSI(Chla) có xu hướng cao hơn trong tháng $5-6$, giảm dần trong tháng 7-9, sau đó tăng nhẹ cho đến tháng 1 năm sau; vùng nước hồ xung quanh phần bờ phía bắc và phía nam có giá $\mathrm{TSI}(\mathrm{Chla})$ cao hơn các vùng nước hồ khác. Nghiên cứu này cho thấy ảnh S2A với độ phân giải không gian phù hợp (lên đến $10 \mathrm{~m}$ ), các kênh phổ được thiết kế hợp lý là loại ảnh có tiềm năng cao để giám sát chất lượng nước các hồ nội địa có diện tích nhỏ, dưới 15 ha như hồ Hoàn Kiếm. Phương pháp nghiên cứu đề xuất trong bài có thể áp dụng vào giám sát lâu dài hiện trạng phú dưỡng của hồ từ ảnh vệ tinh miễn phí $\mathrm{S} 2 \mathrm{~A}$, giúp cho công tác quản lý môi trường thành phố thêm hiệu quả.

Đóng góp của tác giả: Xây dựng ý tưởng nghiên cứu: N.T.P.T., P.Q.V., N.T.T.H.; Lựa chọn phương pháp nghiên cứu: N.T.P.T., P.Q.V., N.T.T.H.; Xử lý số liệu: N.T.P.T., N.T.T.H., N.T.L.; Lấy mẫu: N.T.P.T., P.Q.V., N.T.T.H., N.T.L.; Viết bản thảo bài báo: N.T.P.T., P.Q.V.; Chỉnh sửa bài báo: N.T.P.T., N.T.T.H.

Lời cảm ơn: Nghiên cứu này được thực hiện dưới sự tài trợ của Viện Hàn lâm Khoa học và Công nghệ Việt Nam thông qua đề tài có mã số VAST01.04/19-20. Tập thể tác giả bài báo xin trân trọng cảm ơn sự tài trợ của Viện Hàn lâm Khoa học và Công nghệ Việt Nam, đồng cảm ơn tới ESA và Trung tâm CARGIS, Trường Đại học Khoa học Tự nhiên - ĐHQGHN đã cung cấp ảnh Sentinel và thiết bị đo phổ hiện trường cho nghiên cứu này.

Lời cam đoan: Tập thể tác giả cam đoan bài báo này là công trình nghiên cứu của tập thể tác giả, chưa được công bố ở đâu, không được sao chép từ những nghiên cứu trước đây; không có sự tranh chấp lợi ích trong nhóm tác giả.

\section{Tài liệu tham khảo}

1. Dekker, A.G.; Peters, S.W.M. The use of the Thematic Mapper for the analysis of eutrophic lakes: a case study in the Netherlands. Int. J. Remote Sens. 1993, 14, 799821. https://doi.org/10.1080/01431169308904379. 
2. Cheng, K.S.; Lei, T.C. Reservoir Trophic State Evaluation using Landsat TM Images. JAWRA J. Am. Water Resour. Assoc. 2001, 37, 1321-1334. https://doi.org/10.1111/j.1752-1688.2001.tb03642.x.

3. Tyler, A.N.; Svab, E.; Preston, T.; Présing, M.; Kovács, W.A. Remote sensing of the water quality of shallow lakes: A mixture modelling approach to quantifying phytoplankton in water characterized by high-suspended sediment. Int. J. Remote Sens. 2006, 27, 1521-1537. https://doi.org/10.1080/01431160500419311.

4. Chang, N.B.; Imen, S.; Vannah, B. Remote sensing for monitoring surface water quality status and ecosystem state in relation to the nutrient cycle: a 40-year perspective. Crit. Rev. Env. Sci. Technol. 2015, 45, 101-166. https://doi.org/10.1080/10643389.2013.829981.

5. Jensen, J.R. Remote sensing of the environment: an earth resource perspective: Pearson Prentice Hall. Upper Saddle River, NJ, USA, 2000.

6. Leng, R. The impacts of cultural eutrophication on lakes: A review of damages and nutrient control measures. Writing 2009, 20, 33-39.

7. Le Hung Anh. Untersuchungen zur Verwertung der Biomasse in Landwirtschaft und Gartenbau Vietnams unter besonderer Berücksichtigung der Kompostierung. Humboldt University in Berlin, Dissertation Verlag, ISBN 3-89825-571-9, 2002.

8. Thủy, D.T.; Cường, H.T.; Kim, D.D. Biến động hàm lượng độc tố microcystin trong môi trường nước hồ Hoàn Kiếm. Tạp chi sinh học 2012, 34, 94-98. https://doi.org/10.15625/0866-7160/v34n1.675.

9. Carlson, R.E. A trophic state index for lakes. Limnol. Oceanogr. 1977, 22, 361-369. https://doi.org/10.4319/1o.1977.22.2.0361.

10. Håkanson, L.; Bryhn, A.C.; Hytteborn, J.K. On the issue of limiting nutrient and predictions of cyanobacteria in aquatic systems. Sci. Total Environ. 2007, 379, 89108. https://doi.org/10.1016/j.scitotenv.2007.03.009.

11. Schalles, J.F. Optical remote sensing techniques to estimate phytoplankton chlorophyll-aconcentrations in coastal. In Remote sensing of aquatic coastal ecosystem processes (pp. 27-79). Springer, Dordrecht, 2006.

12. Gholizadeh, M.H.; Melesse, A.M.; Reddi, L. A comprehensive review on water quality parameters estimation using remote sensing techniques. Sensors 2016, 16, 1298. https://doi.org/10.3390/s16081298.

13. Sudheer, K.P.; Chaubey, I.; Garg, V. Lake water quality assessment from Landsat thematic mapper data using neural network: an approach to optimal band combination selection1. JAWRA J. Am. Water Resour. Assoc. 2006, 42, 1683-1695. $\mathrm{https://doi.org/10.1111/j.1752-1688,2006.tb06029.x.}$

14. Turner, D. Remote Sensing of Chlorophyll a Concentrations to Support the Deschutes Basin Lake and Reservoirs TMDLs; Department of Environmental Quality: Portland, OR, USA, 2010.

15. Kloiber, S.M.; Brezonik, P.L.; Bauer, M.E. Application of Landsat imagery to regional-scale assessments of lake clarity. Water Res. 2002, 36, 4330-4340. https://doi.org/10.1016/S0043-1354(02)00146-X.

16. Allan, M.G.; Hicks, B. J.; Brabyn, L. Remote sensing of water quality in the Rotorua lakes. The University of Waikato: Hamilton, New Zealand, 2007.

17. Chipman, J.W.; Olmanson, L.G.; Gitelson, A.A. Remote sensing methods for lake management: A guide for resource managers and decision-makers. North American Lake Management Society, 2009.

18. Menken, K.D.; Brezonik, P.L.; Bauer, M.E. Influence of chlorophyll and colored dissolved organic matter (CDOM) on lake reflectance spectra: Implications for measuring lake properties by remote sensing. Lake and Reservoir Manage. 2006, 22, 179-190. https://doi.org/10.1080/07438140609353895. 
19. Thom, D.T.; Kien, H.T.; Nguyet, V.T.; Kim, D.D. Study on some environmental factors and phytoplankton of Lake Hoan Kiem water before dislodging. The $3^{\text {rd }}$ National Scientific Conference on Ecology and Biological Resources. Hanoi, Vietnam, 2009, pp. 1673.

20. Mobley C.D. Estimation of the remote-sensing reflectance from above-surface $\begin{array}{lllll}\text { measurements. } & \text { Appl. }\end{array}$ https://doi.org/10.1364/AO.38.007442.

21. Barsi, J.A.; Lee, K.; Kvaran, G.; Markham, B.L.; Pedelty, J.A. The spectral response of the Landsat-8 Operational Land Imager. Remote Sens. 2014, 6, 10232-10251. https://doi.org/10.3390/rs61010232.

22. APHA. Standard methods for the examination of water and wastewater analysis. American Public Health Association, Washington DC, 1998.

23. Carlson, R.E.; Simpson, J. A coordinator's guide to volunteer lake monitoring methods. North American Lake Management Society 1996, 96, 305.

24. Ha N.T.T.; Koike K. Integrating satellite imagery and geostatistics of point samples for monitoring spatio-temporal changes of total suspended solids in bay waters: application to Tien Yen Bay (Northern Vietnam). Front. Earth Sci. 2011, 5, 305316. https://doi.org/10.1007/s11707-011-0187-9.

25. Hà, N.T.T.; Cảnh, B.Đ.; Thảo, N.T.P.; Nhị, B.T. Thử nghiệm mô hình hóa sự phân bố không gian của hàm lượng chlorophyll-a và chỉ số trạng thái phú dưỡng nước Hồ Tây sử dụng ảnh Sentinel-2A. VNU Journal of Science: Earth and Environmental Sciences 2016, 32(2S).

\title{
Monitoring the eutrophication level of Lake Hoan Kiem based on the estimated Chlorophyll-a concentration from Sentinel-2A imagery
}

Nguyen Thien Phuong Thao ${ }^{1}$, Pham Quang Vinh ${ }^{2}$, Nguyen Thi Thu Ha ${ }^{1, *}$, Nguyen Thuy Linh ${ }^{1,3}$

${ }^{1}$ Faculty of Geology, VNU University of Science, Vietnam National University, Hanoi, Vietnam; nguyenthienphuongthao_t57@hus.edu.vn

2 Institute of Geography, Vietnam Academy of Science and Technology, Hanoi; pqvinh@ig.vast.vn

${ }^{3}$ Faculty of Geography, VNU University of Science, Vietnam National University, Hanoi, Vietnam; nguyenthuylinh@hus.edu.vn

\begin{abstract}
This study aims to monitor the trophic state index (TSI) of Lake Hoan Kiem using estimated chlorophyll-a concentration (Chla) from Sentinel 2A (S2A) acquired over Hanoi from September 2019 to August 2020. In situ data measured at 50 points over the lake in 5 field-trips shows that Chla ranged from $114.8 \mu \mathrm{g} / \mathrm{L}$ to $700 \mu \mathrm{g} / \mathrm{L}$ and strongly correlated to the ratio of the near-infrared band (band 5) versus the red band (band 4) of $\mathrm{S} 2 \mathrm{~A}$ by an exponential equation $\left(\mathrm{R}^{2}=0.82\right)$. The reasonable error of validation $(29.4 \mu \mathrm{g} / \mathrm{L})$ indicates the equation appropriateness for estimating Chla in the lake water. As a result, estimated TSI(Chla) was harmonized with in situ TSI(Chla) and ranged from 77 to 95, classifying the lake water at the hypertrophic level and confirming a high potential of S2A for monitoring the lake trophic status. In space, TSI(Chla) distributed homogeneously and accumulated little in the areas along the northern and southern shores. Estimated TSI(Chla) was little changed by seasons, at the higher level in early summer (June) and early winter (from October to December). To better monitor TSI(Chla), other sensors such as Landsat 8 and Sentinel 2B should be investigated and exploited.
\end{abstract}

Keywords: The lake trophic state; Ho Guom; Chlorophyll-a; TSI; Sentinel 2A. 